\title{
TERT aberrancies: a screening tool for malignancy in follicular thyroid tumours
}

\author{
Johan O Paulsson', Ninni Mu1, Ivan Shabo²,3, Na Wang', Jan Zedenius,2,3, Catharina Larsson' \\ and C Christofer Juhlin'1
}

'Department of Oncology-Pathology, Karolinska Institutet, Karolinska University Hospital CCK, Stockholm, Sweden 2Department of Molecular Medicine and Surgery, Karolinska Institutet, Karolinska University Hospital, Stockholm, Sweden 3Department of Breast, Endocrine Tumours and Sarcoma, Karolinska University Hospital, Stockholm, Sweden

Correspondence should be addressed to J O Paulsson: johan.paulsson@ki.se

\begin{abstract}
Telomerase reverse transcriptase (TERT) promoter mutations have been linked to adverse clinical parameters in thyroid cancer, but TERT-expressing tumours are not always mutated. Little is known regarding other TERT-related genetic aberrations. To delineate the role of TERT gene aberrancies in follicular thyroid tumours, 95 follicular carcinomas (FTCs), 43 follicular adenomas (FTAs) and 33 follicular tumours of uncertain malignant potential (FT-UMPs) were collected. The tumours were assayed for TERT expression, TERT promoter mutations, TERT promoter hypermethylation and TERT gene copy number (CN) alterations and the results were compared to clinical parameters. Cases with mutation, detectable mRNA expression, CN gain or hypermethylation were classified as TERT aberrant, and these aberrancies were regularly found in FTC and FT-UMP but uncommonly found in FTA. In total, 59\% FTCs and 63\% FT-UMPs exhibited one or more of these TERT gene aberrancies. Moreover, 24 out of 28 FTCs (86\%) with TERT expression displayed an evident TERT gene aberration, and statistics showed an increased risk for relapse in FTCs with TERT expression, CN gain or hypermethylation. We conclude that TERT expression in follicular thyroid tumours is coupled to promoter mutations, CN gain and increased promoter methylation. The molecular similarities regarding TERT aberrations between the FTC and FT-UMP groups indicate that a significant subset of FT-UMP cases may display future recurrences. TERT aberrancies are thus promising as future additional markers for determining malignant potential of follicular thyroid tumours.
\end{abstract}

\section{Introduction}

The telomerase enzyme consists of a protein component with reverse transcriptase activity, encoded by the telomerase reverse transcriptase (TERT) gene (Cong et al. 2002). Telomerase activation is a hallmark of malignant tumours, providing immortalisation through the maintenance of telomere length through numerous cell divisions (Bodnar et al. 1998). Telomerase is rarely activated in normal cells; however, it is detectable in 90\% of human malignancies (Shay \& Bacchetti 1997, Daniel et al. 2012). One important underlying mechanism of telomerase activation constitutes of hot spot mutations (denoted C228T and C250T) in the TERT promoter, which has been shown to upregulate TERT mRNA expression by creating binding motifs for the transcription factor ETS2 
(Huang et al. 2013, Liu et al. 2016). The occurrence of TERT mutations and mRNA expression is in turn strongly associated to telomerase activity (Huang et al. 2015). The two recurrent TERT mutations have been detected in several human malignancies (Vinagre et al. 2013) and recently they were also detected in follicular-cell-derived thyroid malignancies (Landa et al. 2013, Liu et al. 2013a, 2014). These mutations have been intimately linked to aggressive properties in thyroid cancer and has also been suggested as a potential biomarker to aid in prognosis (Liu et al. 2013b, 2014, Melo et al. 2014, Wang et al. 2014, Liu \& Xing 2016).

The mechanisms for induction of TERT are not completely understood, and in a subset of cases, it cannot be explained by a mutation in the TERT promoter (Wang et al. 2014). Previous studies in other tumour types have demonstrated that other aberrancies such as copy number $(\mathrm{CN})$ gain and promoter hypermethylation are associated with increased TERT expression (Guilleret et al. 2002, Cao et al. 2008); however, these alternative mechanisms of TERT upregulation have not yet been extensively studied in thyroid cancer.

Although papillary thyroid carcinoma (PTC) is the most common malignant thyroid tumour, follicular thyroid adenomas (FTAs) and carcinomas (FTCs) are collectively the most common encountered neoplasms in the thyroid gland (DeLellis 2004, Lloyd et al. 2017). They constitute a challenge to the practising pathologist, because the tumours need to display invasive behaviour histopathologically to obtain an FTC diagnosis (DeLellis 2004, Lloyd et al. 2017). If tumours are without capsular and vascular invasion, a diagnosis of follicular thyroid adenoma is put forward (Lloyd et al. 2017). However, to rely solely on histopathological characteristics is sometimes not adequate, and small subsets of seemingly benign FTAs do recur as full-blown malignant tumours (Wang et al. 2014). As regular FTAs are not believed to progress into FTCs, these cases are almost certainly originally misclassified - thereby pinpointing the inability of histopathology to accurately identify malignant potential in every case (Lloyd et al. 2017). To improve the identification of follicular tumours with malignant potential, adjunct algorithms based on Ki-67 proliferation index have been suggested, in which FTAs with proliferation counts above $5 \%$ demand more vigilant follow-up (DeLellis 2004). The World Health Organisation (WHO) 2017 guidelines subsequently introduced the subclassification 'follicular tumour of uncertain malignant potential' (FT-UMP) (Lloyd et al. 2017). An FT-UMP is defined as an 'encapsulated or well-circumscribed tumour composed of well-differentiated follicular cells with no nuclear features of PTC and with questionable capsular or vascular invasion'. This entity is synonymous to the term 'atypical FTA' (AFTA), a term previously used in publications from our group and the terminology presently included in the Swedish national healthcare guidelines for thyroid cancer (Wang et al. 2014). At our institution, patients with FT-UMPs are regularly followed by the endocrine surgeon for 6 months, and then discharged to the primary healthcare system for follow-up with a general practitioner (Hennings et al. 2012).

There is currently a lack of knowledge of molecular alterations of the TERT gene locus that can have a possible effect on the TERT expression in follicular tumours. To investigate this question, we determined mutational status, promoter methylation density and TERT CN alterations in comparison to TERT gene expression. Through clinical follow-up, we also investigated if these alternative molecular aberrancies could be used to aid in the diagnosis and prognostication of FTC.

\section{Materials and methods}

\section{Tumour samples and patient information}

Informed consent was collected before the study was carried out and the study was approved by the Regional Ethical Review Board (Etikprövningsnämnden, Stockholm). The samples were collected from the Karolinska University Hospital Biobank from 1986 to 2017 and verified by an experienced endocrine pathologist. The study included 171 tumours from patients with follicular thyroid tumours. All FTCs were reclassified from the WHO 2004 edition (DeLellis 2004) to the updated WHO 2017 (Lloyd et al. 2017) criteria. In total, 95 cases were diagnosed with FTC including 40 minimally invasive (miFTC), 13 encapsulated angioinvasive (eaiFTC) and 41 widely invasive (wiFTC) according to the WHO 2017 criteria (Lloyd et al. 2017) where data were available. In total, 33 patients displayed FT-UMP and 43 were diagnosed with FTA. One FT-UMP was excluded due to a co-existing PTC. Twelve samples of multi-nodular goitre (MNG) were included as non-tumourous references; these cases were collected from nine female patients and three male patients with a mean age at surgery of 50 years (range, 20-74). No case displayed concurrent Hashimoto thyroiditis, and the median follow-up time was 12 years without any subsequent diagnosis of thyroid carcinoma.

Clinical data for all tumour cases was collected from the patients' medical records at Karolinska University 
Hospital, Solna, Sweden. The clinical variables included age at the time of surgery, gender, Ki-67 index, tumour size, T stadium, type of surgery, radioiodine treatment and dose, as well as time to disease recurrence and diseasespecific death. For subsets of the cases, clinical data have been previously published (Wang et al. 2014). For subsets of cases, parts of the clinical information were unavailable, in addition, not all cases were informative for each genetic analysis due to technical issues.

\section{Extraction of genomic DNA and RNA}

DNA was isolated from fresh frozen tissue using DNeasy Blood and Tissue Kit (Qiagen) and total RNA was isolated from fresh frozen tissue using mirVana miRNA Isolation Kit, with phenol (Invitrogen) according to manufacturer's protocol. Concentrations were determined using the Nanodrop technology (Nanodrop Technologies, Wilmington, DE, USA).

\section{Expression analyses}

Total RNA was used for cDNA synthesis using HighCapacity cDNA Reverse Transcription Kit (Applied Biosystems). Quantitative real-time PCR (qRT-PCR) was performed in 65 FTCs, 23 FT-UMPs and 43 FTAs using ABI 7900HT RT-PCR System and TaqMan Gene expression assays (Applied Biosystems, Hs00972656_m1) to investigate TERT expression levels. The $18 \mathrm{~S}$ rRNA expression was used as a housekeeping gene reference (Applied Biosystems, Hs99999901_s1) as previously described (Liu et al. 2014). Samples were run in triplicates, and the relative expression was calculated as $2^{-\Delta C T}$.

\section{Sanger sequencing}

Sequencing was performed in 66 FTCs and 43 FTAs. One FTC and one FTA failed sequencing due to poor quality of the DNA. For the remaining 29 FTCs and 32 FT-UMPs, sequencing data were already available from routine Sanger sequencing in the clinic as well as from a previous study (Wang et al. 2014). The targeted region was amplified from 50 to $100 \mathrm{ng}$ DNA under the following PCR conditions: $8 \mathrm{~min}$ at $95^{\circ} \mathrm{C} ; 2 \mathrm{~min}$ at $62^{\circ} \mathrm{C} ; 40$ cycles of $\left(2.5 \mathrm{~min}\right.$ at $72^{\circ} \mathrm{C}$, $15 \mathrm{~s}$ at $95^{\circ} \mathrm{C}$ and $1 \mathrm{~min}$ at $62^{\circ} \mathrm{C}$ ) and a final elongation step for $7 \mathrm{~min}$ at $72^{\circ} \mathrm{C}$. The protocol is optimized for regions C228T and C250T with validated TERT primers, forward primer 5'-CACCCGTCCTGCCCCTTCACCTT-3' and reverse primer 5'-GGCTTCCCACGTGCGCAGCAGGA-3' (Wang et al. 2014). The PCR product was cleaned-up using ExoSap-IT (Applied Biosystems) and sequenced via KIGene Core Facility for TERT promoter mutations using conventional Sanger sequencing with the same primer pair. Mutational calling including visual inspection of each chromatogram as well as using CodonCode Aligner (CodonCode Cooperation, Centerville, MA, USA).

\section{Pyrosequencing}

DNA from 77 FTC, 25 FT-UMP and 42 FTA were used for bisulphite conversion with the EpiTect Fast Bisulphite Conversion Kit (Qiagen). The bisulphite-converted DNA was amplified using Qiagen PCR kit for 45 cycles of $30 \mathrm{~s}$ at $94^{\circ} \mathrm{C}, 30 \mathrm{~s}$ at $58^{\circ} \mathrm{C}$ and $30 \mathrm{~s}$ at $72^{\circ} \mathrm{C}$. The targeted region is located between -578 and $-541 \mathrm{bp}$, named Region A as previously described (Wang et al. 2016). CpG methylation was quantified at eight sites within Region A using PyroMark Q24 with Pyromark Gold Q24 reagents, and the data were analysed using Pyro Mark Q24 software (Qiagen). A methylation index (MetI) was calculated for each individual sample. Twelve cases of MNGs were similarly analysed for comparison.

\section{$\mathrm{CN}$ alterations}

Five nanograms of genomic DNA were used to analyse CN aberrations of the TERT gene in 77 FTC, 19 FT-UMP and 43 FTA. The samples were analysed using the TaqMan assays (Applied Biosystems), Hs 01237576_CN for TERT and Hs 4403326_C for the RNaseP gene, which was used as endogenous control. The $\mathrm{CN}$ was calculated using CopyCaller v.2.1 (Applied Biosystems). Two cases of MNG were included as diploid controls. $\mathrm{CN}$ gain was defined as three or more copies and CN loss as one copy or less.

\section{Statistical analyses}

Categorical and binary variables were presented as number (n) and proportion (\%) of cases, respectively. TERT expression, mutation, $\mathrm{CN}$ and hypermethylation were defined as yes or no. Chi-square and Fisher's exact test were used to compare categorical variable differences in TERT expression, mutations, CN gain, hypermethylation and TERT aberrancies combined between the different tumour subtypes. Kaplan-Meier survival analysis was used to plot relapse-free survival time among FTC patients with and without TERT aberrancies and log-rank test was used to calculate significance. Relapse-free survival time was defined as number of months before clinical or histopathological evidence of relapsing disease (local 
recurrences or metastases). Cox regression was used to analyse the association of covariates to relapse in both univariate and multivariate analyses. Since there exist probable biological associations between one or several of the factors corrected for (especially age and tumour stage) and the end-point parameter (relapse), we included multivariate analysis also for associations deemed not significant by univariate analysis. Mann-Whitney $U$ test, chi-square and Fisher's exact test were used to compare aberrancies to clinicopathological variables. $P$ values $<0.05$ were considered as significant, except for all analyses performed in Supplementary Table 1 (see section on supplementary data given at the end of this article). As numerous correlations were sought, although the vast majority of parameters were indeed dependent variables, we lowered the threshold of statistical significance to $P<0.01$ to avoid the bias of a potential type I error while still not increasing the risk of a type II error significantly. All statistical calculations were performed in SPSS Statistics 24 software (IBM).

\section{Results}

\section{Patient characteristics}

Clinical characteristics for all patients with follicular thyroid tumours included in the study are described in Table 1. According to the WHO 2017 guidelines, 40 FTCs were miFTC, 13 were eaiFTC and 41 were wiFTC. The mean follow-up time was 99.5 months (7-373). At the time of follow-up, 20 patients had spread disease and 16 patients had died from FTC. One of the 33 FT-UMP patients had a relapse and died from FTC. This patient was operated at age 63 years and was later diagnosed with metastatic FTC and died at age 74 years, as previously reported (Wang et al. 2014). The tumour capsule of the original lesion was submitted entirely for histological evaluation, which is standardized routine when an FT-UMP is diagnosed in our institution, and hence, there should be little risk of an evident focus displaying vascular or capsular invasion being overlooked at the time of diagnosis. As there were no apparent macroscopical or microscopical foci with capsular or lymphovascular invasion, a diagnosis of FTC could be rejected. No relapses were recorded among the 43 FTA cases.

\section{TERT expression is common in FTC and FT-UMP but not in FTA}

Based on qRT-PCR of informative tumours, 28/65 (43\%) FTCs, 9/23 (39\%) FT-UMPs and 6/43 (14\%) FTAs showed
TERT expression. Based on FTC subtypes according to WHO 2017, expression was observed in 10 out of 22 miFTCs (45\%), 2 out of 7 eaiFTCs (29\%) and 16 out of 36 wiFTCs (44\%) (Supplementary Table 1). There was no significant difference between the proportions of TERT expressing tumours in the FTC and FT-UMP groups $(P=0.742)$. In contrast, significantly more FT-UMPs than FTAs that displayed TERT expression $(P=0.020)$. The results are summarized in Table 2 .

\section{TERT promoter mutation frequency}

TERT promoter mutation frequencies were determined based on Sanger sequencing. In total, mutations were present in 19/94 (20\%) of FTCs, 6/32 (19\%) of FT-UMPs and $0 / 42(0 \%)$ of FTAs. There was no statistical difference in mutation frequency between the FTC and FT-UMP groups $(P=0.858)$ (Table 2). However, no FTA displayed a TERT promoter mutation, and the difference between FT-UMPs and FTAs was significant $(P=0.005)$ (Table 2).

\section{TERT promoter hypermethylation}

To investigate novel molecular aberrancies linked to TERT, we performed pyrosequencing targeting an established part of the TERT gene promoter (Region A) in which hypermethylation (but not hypomethylation) has been shown to be linked to TERT expression (Dessain et al. 2000, Castelo-Branco et al. 2013). A MetI was calculated for each sample analysed as a mean of the eight CpG sites. The median MetI was 13\% in FTC, 11\% in FT-UMP, $8 \%$ in FTA and 6\% in MNG. The MetI was higher in FTCs and FT-UMP compared to FTAs $(P<0.001$ and $P=0.045$, respectively). The MetI is illustrated in Supplementary Fig. 1. The highest MetI in an FTA was $18 \%$, and therefore, MetI above $18 \%$ was labelled as hypermethylated in the following statistical calculations. The frequency of hypermethylated cases was then compared in the tumour entities. The frequency of hypermethylated cases was 25/77 (32\%) in FTC and 3/25 (12\%) in FT-UMP (Table 2).

\section{TERT CN alterations}

TERT gene $\mathrm{CN}$ were determined with TaqMan $\mathrm{CN}$ assay. In FTC, 5/77 (6\%) showed a CN loss and 6/77 (8\%) cases showed three or more copies of the TERT gene (Table 2). The CN aberration was similar in FT-UMP with 5/19 (26\%) cases displaying either gain $(n=4)$ or loss $(n=1)$ (Table 2$)$. 
Table 1 Clinical characteristics of cases with FTC, FT-UMP and FTA in the study.

\begin{tabular}{|c|c|c|c|c|c|c|}
\hline \multirow[b]{2}{*}{ Parameter } & \multicolumn{2}{|c|}{ FTC cases } & \multicolumn{2}{|c|}{ FT-UMP cases } & \multicolumn{2}{|c|}{ FTA cases } \\
\hline & Data & Observation & Data & Observation & Data & Observation \\
\hline Age at surgery, $n$ & 95 & & 33 & & 43 & \\
\hline Mean (min-max) years & & $56(11-91)$ & & $49.8(25-76)$ & & $50.5(25-87)$ \\
\hline Gender, $n$ & 95 & & 33 & & 43 & \\
\hline Female, $n(\%)$ & & $59(62)$ & & $28(85)$ & & $31(72)$ \\
\hline Type of surgery, $n$ & 67 & & 33 & & 43 & \\
\hline Total thyroidectomy, $n(\%)$ & & $62(93)$ & & $5(15)$ & & $1(2)$ \\
\hline Hemithyroidectomy, n (\%) & & $5(7)$ & & $28(85)$ & & $42(98)$ \\
\hline Radioiodine treatment, $n$ & 68 & & 33 & & 43 & \\
\hline Received, $n(\%)$ & & $60(88)$ & & $1(3)$ & & $0(0)$ \\
\hline Radioiodine dose, $n$ & 60 & & & & & \\
\hline Median (range) (Mbq) & & $3700(1100-7400)$ & & - & & - \\
\hline Tumour size, $n$ & 92 & & 33 & & 43 & \\
\hline Mean (min-max) (mm) & & $42.8(15-100)$ & & $36.0(15-80)$ & & $31.9(14-60)$ \\
\hline T-stage, $n$ & 94 & & & & & \\
\hline pT1, n (\%) & & $12(13)$ & & - & & - \\
\hline pT2, $n(\%)$ & & $27(29)$ & & - & & - \\
\hline pT3, $n(\%)$ & & $54(57)$ & & - & & - \\
\hline $\mathrm{pT} 4, n(\%)$ & & $1(1)$ & & - & & - \\
\hline Hürthle cell, $n$ & & & & & 43 & \\
\hline Yes & $n=92$ & $n=20(22 \%)$ & $n=33$ & $n=9(28 \%)$ & & $n=15(35 \%)$ \\
\hline Ki-67 index, $n$ & & & & & 12 & \\
\hline Mean (min-max) & $n=64$ & $6 \%(1-32 \%)$ & $n=16$ & $7 \%(1-17 \%)$ & & $2 \%(0-3 \%)$ \\
\hline Extrathyroidal growth & & & & & & \\
\hline Present & $n=94$ & $n=11(12 \%)$ & & - & & - \\
\hline Lymph node spread & & & & & & \\
\hline Present & $n=47$ & $n=5(11 \%)$ & & - & & - \\
\hline Subtype (WHO 2004) & & & & & & \\
\hline miFTC & $n=94$ & $n=38(40 \%)$ & & - & & - \\
\hline wiFTC & & $n=56(60 \%)$ & & - & & - \\
\hline Subtype (WHO 2017) & & & & & & \\
\hline MiFTC & $n=94$ & $n=40(43 \%)$ & & - & & - \\
\hline eaiFTC & & $n=13(13 \%)$ & & - & & - \\
\hline wiFTC & & $n=41(44 \%)$ & & - & & - \\
\hline Follow-up time & & & & & $n=43$ & \\
\hline Mean (min-max) (months) & $n=95$ & $99.5(7-373)$ & $n=33$ & $144.6(9-336)$ & & $69.8(23-140)$ \\
\hline Survival & & & & & $n=43$ & \\
\hline Alive & $n=95$ & $n=67(70 \%)$ & $n=33$ & $n=28(85 \%)$ & & $n=40(93 \%)$ \\
\hline Dead of other cause, $n(\%)$ & & $12(13)$ & & $4(12)$ & & $3(7)$ \\
\hline Dead of disease, $n(\%)$ & & $16(17)$ & & $1(3)$ & & $0(0)$ \\
\hline Outcome & & & & & $n=43$ & \\
\hline Disease-free & $n=89$ & $n=69(78 \%)$ & $n=33$ & $n=32(97 \%)$ & & $n=43(100 \%)$ \\
\hline Spread disease, $n(\%)$ & & $20(22)$ & & $1(3)$ & & $0(0)$ \\
\hline
\end{tabular}

eaiFTC, encapsulated angioinvasive FTC; FTA, follicular thyroid adenoma; FTC, follicular thyroid carcinoma; FT-UMP, follicular tumour of uncertain malignant potential; miFTC, minimally invasive FTC; wiFTC, widely invasive.

\section{TERT aberrancies combined}

In Table 2, the TERT aberrancies (promoter mutation, detectablemRNAexpression, CNgainorhypermethylation) were combined to compare the molecular differences and similarities between the tumour entities. In FTC, 43/73 (59\%) cases had at least one TERT aberrancy, which was similar to FT-UMP where 12/19 (63\%) cases displayed an aberrancy. In FTA, 6/42 (14\%) cases were TERT aberrant, which was significantly less than in the FT-UMP group $(P<0.001)$.

http://erc.endocrinology-journals.org https://doi.org/10.1530/ERC-18-0050

\section{TERT expressing FTC and FT-UMP cases with} molecular TERT aberrancies

A summary of all TERT expressing FTCs and FT-UMPs with regards to genetic and clinical parameters is detailed in Supplementary Table 2. In total, 28 cases of FTC exhibited TERT mRNA expression, 13 of these tumours harboured a TERT mutation. However, when mutation, $\mathrm{CN}$ gain and hypermethylation were combined, 24 of the 28 TERT expressing cases displayed a TERT aberrancy in any of the categories mentioned earlier. TERT expression in FTC 


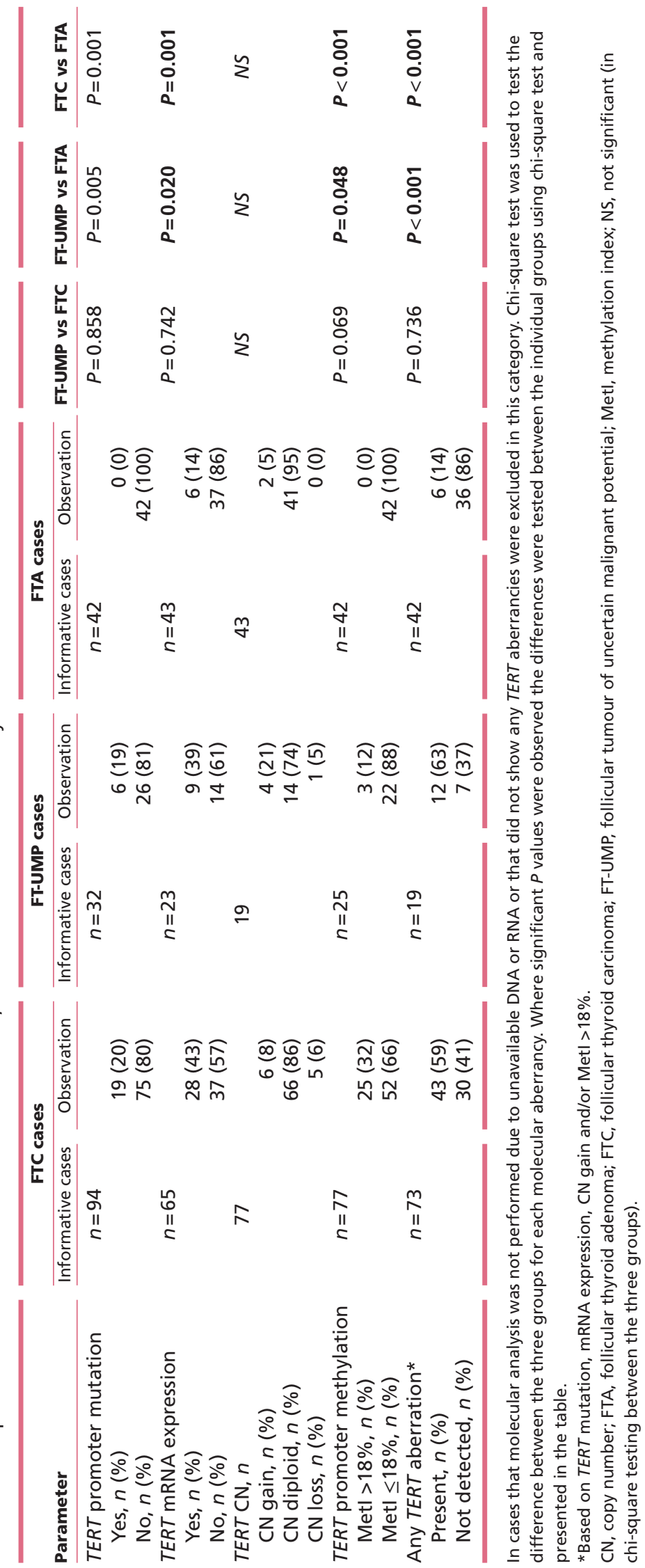


was significantly associated to TERT mutation, $\mathrm{CN}$ gain and hypermethylation $(P<0.001, P=0.023$ and $P<0.001$, respectively). In the FT-UMP group, 9 out of 23 cases (39\%) exhibited TERT mRNA expression. Out of these, eight cases (89\%) demonstrated a TERT gene aberrancy.

\section{TERT molecular aberrancies and clinicopathological parameters}

Clinical and histopathological parameters in FTC were tested for association to TERT aberrancies using univariate analyses. TERT expression, mutation, hypermethylation and 'TERT aberrant' were significantly associated to 'age at surgery' (Supplementary Table 1). All calculations and $P$ values are specified in Supplementary Table 1.

\section{TERT expression, hypermethylation and $\mathrm{CN}$ gain are associated with recurrence in FTC}

To investigate if molecular TERT aberrancies could be used as a predictor for a worse clinical outcome, a log-rank test was performed for each molecular aberrancy as well as for all aberrancies combined (TERT aberrancies) and visualised in Kaplan-Meier survival curves (Fig. 1). Cox regression was then used to calculate hazard ratio (HR) after adjusting for covariates; older age, male gender, T-stage and FTC subtype. In the TERT expression group ( $n=28), 11$ events occurred comparing to five events in the group with absent TERT expression $(n=37)$. Time to recurrence was shorter in the TERT expression group ( $P=0.001$, Fig. 1A). HR for relapse in cases with TERT expression relative to absent TERT expression was 4.267 (95\% CI, 1.069-17.033, $P=0.040$ ) (Table 3). In the TERT mutation group ( $n=19), 8$ events occurred compared to 14 events in the WT group $(n=75)$ (Table 3$)$. Time to recurrence was shorter among TERT-mutated cases and in cases with any TERT aberrancy $(P=0.040$ and $P=0.043$, Fig. $1 \mathrm{~B}$ and $\mathrm{E}$, respectively). No significant changes were seen for cases with hypermethylation or $\mathrm{CN}$ gain (Fig. 1C and $\mathrm{D}$, respectively).

\section{TERT aberrancies as diagnostic and prognostic markers}

The sensitivity, specificity as well as positive and negative predictive values (PPVs and NPVs, respectively) were calculated for all four individual TERT aberrant parameters (promoter mutation, mRNA expression, $\mathrm{CN}$ gain and methylation) as well as all parameters combined for the
A TERT MRNA expression

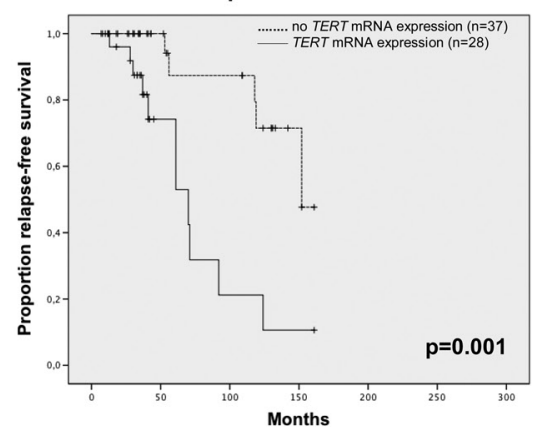

C TERT promoter Met|>18\%

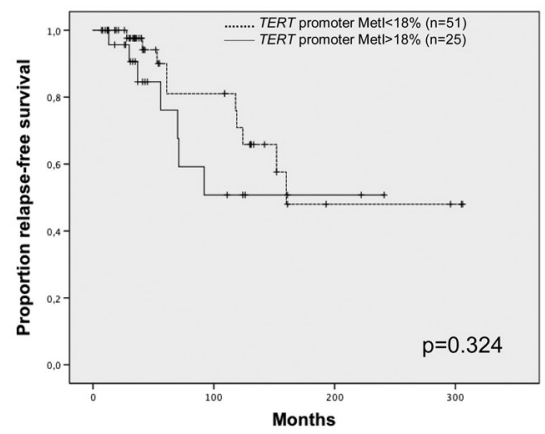

B TERT promoter mutation

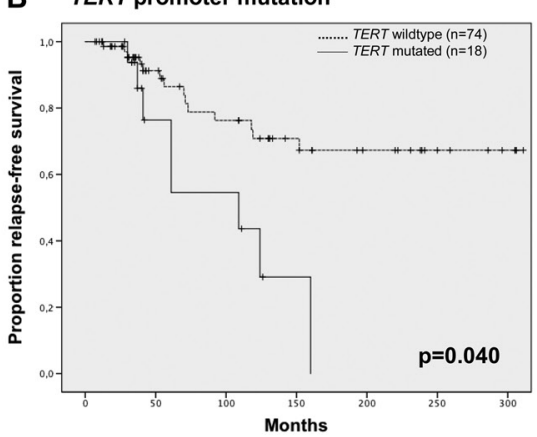

D TERT copy number gain

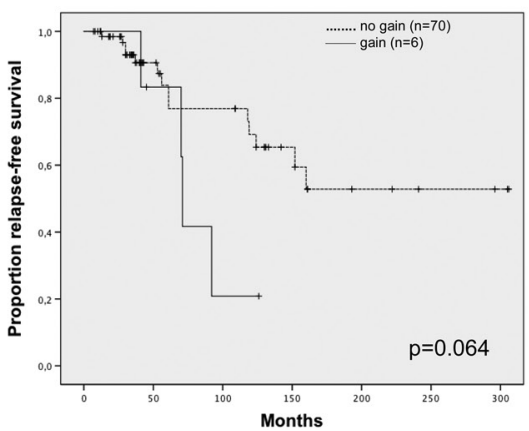

E TERT aberrant

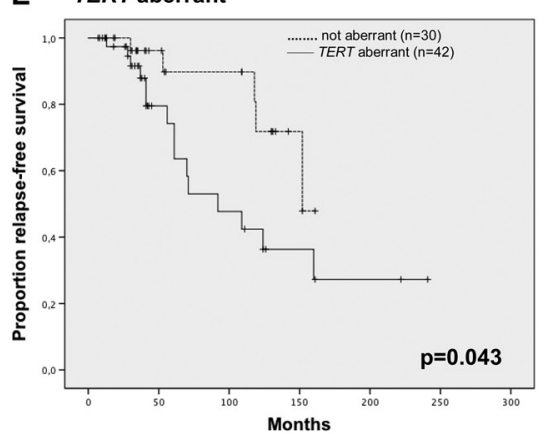

Figure 1

Kaplan-Meier survival curves in follicular thyroid carcinoma (FTC) comparing time to recurrence in telomerase reverse transcriptase (TERT) aberrant cases with TERT WT cases. (A) TERT expression, (B) TERT mutation, (C) TERT promoter hypermethylation, (D) TERT copy number gain, (E) All aberrancies combined. $P$ values were calculated using log-rank test. Significant $P$ values are in bold. 
Table 3 Hazard ratios for variables associated with relapse in FTC.

\begin{tabular}{|c|c|c|c|c|c|c|}
\hline \multirow[b]{2}{*}{ Covariate } & \multicolumn{3}{|c|}{ Univariate analyses } & \multicolumn{3}{|c|}{ Multivariate analyses } \\
\hline & Coefficient & $\mathrm{HR}(95 \% \mathrm{Cl})$ & $P$ value & Coefficient & $\mathrm{HR}(95 \% \mathrm{Cl})$ & $P$ value \\
\hline TERT mRNA expression & 1.718 & $5.574(1.886-16.470)$ & 0.002 & 1.451 & 4.267 (1.069-17.033) & 0.040 \\
\hline Older age & 0.051 & $1.052(1.023-1.082)$ & $<0.001$ & 0.037 & $1.038(0.989-1.089)$ & 0.132 \\
\hline Male gender & 1.012 & $2.752(1.174-6.453)$ & 0.020 & 0.901 & $2.462(0.759-7.986)$ & 0.133 \\
\hline T-stage & 1.277 & 3.587 (1.198-10.741) & 0.022 & 0.746 & $2.108(0.354-12.565)$ & 0.413 \\
\hline mi/eai/wi (WHO 2017) & 1.082 & $2.950(1.602-5.431)$ & 0.001 & 0.347 & $1.415(0.539-3.714)$ & 0.481 \\
\hline TERT promoter mutation & 1.219 & $3.384(1.397-8.197)$ & 0.007 & 0.004 & $1.004(0.370-2.724)$ & 0.994 \\
\hline Older age & & & & 0.068 & $1.071(1.029-1.114)$ & 0.001 \\
\hline Male gender & & & & 0.732 & $2.080(0.801-5.399)$ & 0.132 \\
\hline T-stage & & & & 1.051 & $2.860(0.881-9.287)$ & 0.080 \\
\hline mi/eai/wi (WHO 2017) & & & & 0.408 & $1.503(0.745-3.034)$ & 0.255 \\
\hline TERT promoter Metl >18\% & 0.481 & $1.618(0.615-4.262)$ & 0.330 & 1.454 & $4.281(1.328-13.801)$ & 0.015 \\
\hline Older age & & & & 0.054 & $1.056(1.012-1.101)$ & 0.011 \\
\hline Male gender & & & & 0.509 & $1.663(0.566-4.882)$ & 0.355 \\
\hline T-stage & & & & 2.359 & $10.584(1.856-60.338)$ & 0.008 \\
\hline mi/eai/wi (WHO 2017) & & & & 0.710 & $2.033(0.830-4.984)$ & 0.121 \\
\hline TERT CN gain & 1.033 & $2.811(0.893-8.851)$ & 0.077 & 1.347 & $3.847(1.044-14.170)$ & 0.043 \\
\hline Older age & & & & 0.055 & $1.057(1.016-1.099)$ & 0.006 \\
\hline Male gender & & & & 0.521 & $1.684(0.570-4.977)$ & 0.345 \\
\hline T-stage & & & & 1.129 & $3.094(0.679-14.095)$ & 0.144 \\
\hline mi/eai/wi (WHO 2017) & & & & 0.854 & $2.350(0.985-5.602)$ & 0.054 \\
\hline TERT aberrant* & 1.234 & 3.435 (1.131-10.437) & 0.029 & 1.014 & $2.757(0.822-9.239)$ & 0.100 \\
\hline Older age & & & & 0.049 & $1.050(1.007-1.096)$ & 0.022 \\
\hline Male gender & & & & 0.685 & $1.983(0.689-5.712)$ & 0.205 \\
\hline T-stage & & & & 1.350 & $3.857(0.978-15.208)$ & 0.054 \\
\hline mi/eai/wi (WHO 2017) & & & & 0.253 & $1.288(0.589-2.818)$ & 0.526 \\
\hline
\end{tabular}

Univariate and multivariate analyses were calculated using Cox regression analysis. Significant $P$ values are in bold. *Based on TERT mutation, mRNA expression, CN gain and/or Metl >18\%.

eai, encapsulated angioinvasive FTC; FTC, follicular thyroid carcinoma; HR, hazard ratio; Metl, methylation index; mi, minimally invasive FTC; wi, widely invasive FTC.

distinction between FTC/FT-UMA vs FTA and for the distinction between relapsing vs relapse-free FTC cases (Table 4). In general, high specificity and PPVs were obtained for individual as well as combined TERT aberrant parameters, suggesting that the detection of any such a genetic event would imply high risk of FTC or FT-UMP as opposed to FTA in a diagnostic setting. Moreover, when comparing relapsing and non-relapsing FTCs, high sensitivity and high NPV was seen for TERT aberrancies combined, suggesting that absence of these genetic events in a histopathologically diagnosed FTC would suggest a lower risk of future recurrences.

\section{Discussion}

FTCs have been previously shown to display TERT expression (Saji et al. 1999), although the underlying molecular mechanisms driving this phenomenon have been obscure. The finding of TERT promoter mutations in subsets of FTCs helped elucidate a large subset of FTCs with TERT expression, although a large number of tumours with evident expression were devoid of promoter mutations. Recent studies in other forms of thyroid cancers have identified evident methylation and CN gain in TERT (Capezzone et al. 2008, Jendrzejewski et al. 2011, Wang et al. 2016). In this study, we show how aberrant TERT promoter methylation and CN gains of the TERT gene occurs in FTC. In total, 24 out of 28 FTCs (86\%) with TERT expression displayed an evident TERT gene aberration, thereby giving new insights regarding the underlying genetic events driving TERT upregulation in follicular thyroid cancer.

TERT expression has previously been associated to malignant phenotype in various tumours, including thyroid cancer (Foukakis et al. 2007). In this study, FTCs with TERT expression alone significantly predicted an older age at surgery, high Ki-67 index, persistent/recurrent disease as well as death of disease. Other molecular aberrancies (promoter mutation, hypermethylation, CN gain or all combined) did not display the same amount of significant associations (Supplementary Table 1). These findings corroborate the previous studies regarding TERT expression in malignant thyroid tumours and highlight the possible usage of TERT expression as a predictive marker of worse clinical outcome in FTCs. 
Table 4 TERT aberrancies as a diagnostic and prognostic marker.

\begin{tabular}{|c|c|c|c|c|}
\hline Parameter & Sensitivity (\%) & Specificity (\%) & PPV (\%) & NPV (\%) \\
\hline \multicolumn{5}{|c|}{ TERT aberrancies as a diagnostic marker to distinguish FTC/FT-UMP from FTA } \\
\hline TERT promoter mutation & 20 & 100 & 100 & 29 \\
\hline TERT mRNA expression & 42 & 86 & 86 & 42 \\
\hline TERT CN variation & 17 & 95 & 89 & 34 \\
\hline TERT CN gain & 10 & 95 & 83 & 32 \\
\hline TERT promoter hypermethylation & 27 & 100 & 100 & 36 \\
\hline Any TERT aberration* & 60 & 86 & 90 & 49 \\
\hline \multicolumn{5}{|c|}{ TERT aberrancies as a prognostic marker to distinguish relapsing FTC from relapse-free FTC } \\
\hline TERT promoter mutation & 36 & 86 & 44 & 81 \\
\hline TERT mRNA expression & 69 & 65 & 39 & 86 \\
\hline TERT CN variation & 22 & 88 & 36 & 78 \\
\hline TERT CN gain & 22 & 97 & 67 & 80 \\
\hline TERT promoter hypermethylation & 41 & 69 & 28 & 80 \\
\hline Any TERT aberration* & 79 & 49 & 36 & 87 \\
\hline
\end{tabular}

*Based on TERT mutation, mRNA expression, CN gain and/or Metl $>18 \%$.

CN, copy number; FTA, follicular thyroid adenoma; FTC, follicular thyroid carcinoma; FT-UMP, follicular tumour of uncertain malignant potential; NPV, negative predictive value; PPV, positive predictive value.

FTAs are benign tumours, and in our material, TERT expression was only evident in small subset of cases (14\%). None of the FTAs with detectable TERT expression displayed aberrancies in the TERT gene, and therefore, the exact underlying cause of expression in these cases remains obscure. Moreover, no relapses or metastases were detected at follow-up for these cases, suggesting that they in fact were benign at the time of diagnosis. As lymphocytes are differentiated non-tumourous cells that express telomerase, we reviewed all six FTAs exhibiting TERT expression microscopically. One case displayed prominent intra-tumoural lymphocytic infiltrates as part of a previously diagnosed B-cell chronic lymphocytic leukaemia (B-CLL). All other cases were devoid of visible lymphocytic infiltrates (data not shown). The finding of B-CLL within the tumour could in theory explain the positive TERT expression in this case, as approximately half of all B-CLLs express TERT mRNA (Tchirkov et al. 2004). For the remaining five FTA cases, the reason for TERT expression remains obscure. An additional two FTAs displayed aberrancies of the TERT gene (both with $\mathrm{CN}$ gains). None of these cases displayed TERT expression, suggesting that TERT CN gains not automatically confer augmented expression.

Several studies of follicular thyroid neoplasms have been conducted with the main aim to detect distinguishing markers for the proper detection of malignant potential, including immunohistochemical markers such as Ki-67, Galectin-3, as well as expressional analyses including micro-RNA patterns and more comprehensive DNA mutational panels such as ThyroSeq (Heikkila et al. 2010, Labourier et al. 2015, Maruta et al. 2015, Nikiforov et al. 2015). However, many of these promising markers display somewhat reduced sensitivity and/or specificity, alternatively the sheer number of markers (such as provided through expressional or mutational panels) makes the analyses costly and somewhat dependent on tertiary pathology centres with expertise in molecular pathology to provide an accurate interpretation of the results. TERT promoter mutational screening on the other hand is fairly cheap and easy to perform as well as interpret. In our material, the presence of a TERT promoter mutation was intimately coupled to FTCs and FT-UMPs (AFTAs), providing 100\% specificity and a PPV of $100 \%$ for these entities, since no FTAs with mutation were observed. This highlights the diagnostic properties of TERT promoter mutations in a clinical setting, as the finding of a TERT promoter mutation would imply that an FTA diagnosis could be excluded. Moreover, TERT promoter mutational screening was a fairly strong predictor to rule out future recurrences in FTCs with an estimated NPV of $81 \%$, highlighting the established value of TERT promoter screening as a prognostic tool on postoperative material - as absence of these mutations pinpoints FTCs with a favourable outcome.

In addition to the diagnostic and prognostic roles of TERT promoter mutations, the presence of TERT expression, TERT CN gain or hypermethylation in an FTC was strongly associated to a subsequent relapse after adjusting for covariates in our material, indicating these three parameters as possible adjunct screening tools in the clinical setting to pinpoint 'poor prognosis cases'. Moreover, TERT aberrancies were present in the majority of FTCs (59\%) and FT-UMPs (63\%) compared to FTAs (14\%), providing a PPV of $90 \%$ and an NPV of $49 \%$ towards the two former diagnoses. 
Furthermore, when TERT expression was coupled to an underlying TERT gene anomaly (mutation, $\mathrm{CN}$ gain and/or aberrant methylation), no FTA cases with TERT expression in combination with any aberrancy were detected, providing high specificity towards FTC and FT-UMP (PPV 100\%, NPV 55\%, Table 4). Therefore, a postoperative genetic analysis of tumour material in which TERT expression joined by TERT gene aberrancies is assessed could help pinpoint cases with a possible malignant molecular phenotype, even in the absence of histopathological evidence of malignancy. In our series, this is exemplified in the FT-UMP group, in which the only case with later recurrence displayed TERT expression and CN gain in the primary tumour. Our findings could constitute the basis for further investigations on pre-operative cytology material of follicular thyroid tumours, with the aim to study if TERT aberrancies could be used as a screening marker. More precisely, if TERT expression and an associated TERT aberrancy were detected on a fine-needle biopsy material, the responsible clinician could in theory reject FTA as the likely diagnosis.

Since 2014, all confirmed cases of FT-UMP at our institution have been screened for TERT promoter mutations as part of the routine clinical workup based on the previous findings of TERT mutations as a prognostic marker (Wang et al. 2014). The results for these cases have been discussed at multidisciplinary conferences in which the TERT promoter mutational screening might motivate more aggressive treatment options than the standardized protocol in which patients are routinely followed for 6 months, then discharged as out-patients for the primary health care centres (Hennings et al. 2012). However, the clinicians lack current guidelines to adhere to and hence have to discuss TERT mutation positive FT-UMP patients on a case-by-case basis, and the scientific basis from which to draw conclusions on how to follow or treat these patients is thin. Based on the experiences from this study, FT-UMPs carry genetic resemblance to FTCs from a TERT perspective - not least given the similar frequencies of various TERT aberrancies discovered. This is especially true for TERT promoter mutations and TERT expression, whereas the frequencies of cases with TERT promoter hypermethylation and TERT CN gain varied slightly (but not significantly) between the FTC and FT-UMP groups. In our cohort, a single case with FT-UMP did recur as a bona fide FTC. Therefore, it seems safe to conclude that small subsets of FT-UMPs with TERT alterations might represent full-blown malignant cases not yet displaying histopathological evidence of invasion, and we therefore advocate that FT-UMPs displaying TERT aberrancies should be monitored more carefully from a clinical standpoint.

We conclude that TERT expression is coupled to promoter mutations, $\mathrm{CN}$ gain and promoter hypermethylation in FTCs and FT-UMPs. The similar TERT aberrant profiles in the FTC and FT-UMP groups indicate that a subset of tumours in the latter group may develop future recurrences. TERT expression and associated TERT gene aberrancies could thus be a promising future marker for determining malignant potential in follicular tumours of the thyroid.

\section{Supplementary data}

This is linked to the online version of the paper at https://doi.org/10.1530/ ERC-18-0050.

Declaration of interest

The authors declare that there is no conflict of interest that could be perceived as prejudicing the impartiality of the research reported.

\section{Funding}

This work was supported by the Swedish Cancer Society, The Swedish Society for Medical Research, the Cancer Research Funds of Radiumhemmet, Karolinska Institutet and the Stockholm County Council.

\section{Acknowledgements}

The authors are indebted to $\mathrm{Dr}$ Claes Juhlin, Department of Surgical Sciences, Endocrine Surgery, Akademiska sjukhuset, Uppsala, Sweden for providing assistance with clinical follow-up for a fraction of cases. The authors would also like to thank Lisa Ånfalk at the Department of Pathology-Cytology, Karolinska University Hospital for assistance with collecting the tumour specimens from the Biobank, and Elisabeth Berg, statistician at the Unit for Medical Statistics, Department of Learning, Informatics and Ethics (LIME), Karolinska Institutet, for professional help with the statistical analyses and their interpretation.

\section{References}

Bodnar AG, Ouellette M, Frolkis M, Holt SE, Chiu CP, Morin GB, Harley CB, Shay JW, Lichtsteiner S \& Wright WE 1998 Extension of life-span by introduction of telomerase into normal human cells. Science 279 349-352. (https://doi.org/10.1126/science.279.5349.349)

Cao Y, Bryan TM \& Reddel RR 2008 Increased copy number of the TERT and TERC telomerase subunit genes in cancer cells. Cancer Science 99 1092-1099. (https://doi.org/10.1111/j.1349-7006.2008.00815.x)

Capezzone M, Cantara S, Marchisotta S, Filetti S, De Santi MM, Rossi B, Ronga G, Durante C \& Pacini F 2008 Short telomeres, telomerase reverse transcriptase gene amplification, and increased telomerase activity in the blood of familial papillary thyroid cancer patients. Journal of Clinical Endocrinology and Metabolism 93 3950-3957. (https://doi.org/10.1210/jc.2008-0372)

Castelo-Branco P, Choufani S, Mack S, Gallagher D, Zhang C, Lipman T, Zhukova N, Walker EJ, Martin D, Merino D, et al. 2013 Methylation of the TERT promoter and risk stratification of childhood brain tumours: an integrative genomic and molecular
(2) 2018 Society for Endocrinology Published by Bioscientifica Ltd. Printed in Great Britain 
study. Lancet Oncology 14 534-542. (https://doi.org/10.1016/S14702045(13)70110-4)

Cong YS, Wright WE \& Shay JW 2002 Human telomerase and its regulation. Microbiology and Molecular Biology Reviews 66 407-425. (https://doi.org/10.1128/MMBR.66.3.407-425.2002)

Daniel M, Peek GW \& Tollefsbol TO 2012 Regulation of the human catalytic subunit of telomerase (hTERT). Gene 498 135-146. (https:// doi.org/10.1016/j.gene.2012.01.095)

DeLellis RA 2004 World Health Organization Classification of Tumors: Pathology and Genetics of Tumours of Endocrine Organs. Lyon, France: IARC Press.

Dessain SK, Yu H, Reddel RR, Beijersbergen RL \& Weinberg RA 2000 Methylation of the human telomerase gene $\mathrm{CpG}$ island. Cancer Research 60 537-541.

Foukakis T, Gusnanto A, Au AY, Hoog A, Lui WO, Larsson C, Wallin G \& Zedenius J 2007 A PCR-based expression signature of malignancy in follicular thyroid tumors. Endocrine-Related Cancer 14 381-391. (https://doi.org/10.1677/ERC-06-0023)

Guilleret I, Yan P, Grange F, Braunschweig R, Bosman FT \& Benhattar J 2002 Hypermethylation of the human telomerase catalytic subunit (hTERT) gene correlates with telomerase activity. International Journal of Cancer 101 335-341. (https://doi.org/10.1002/ijc.10593)

Heikkila A, Siironen P, Hagstrom J, Heiskanen I, Sankila R, Louhimo J, Haglund C \& Arola J 2010 Follicular thyroid neoplasm: clinicopathologic features suggesting malignancy. Acta Pathologica, Microbiologica et Immunologica Scandinavica 118 846-854. (https:// doi.org/10.1111/j.1600-0463.2010.02668.x)

Hennings J, the National Taskforce via the Regional Cancer Centre West 2012 Sköldkörtelcancer Nationellt Vårdprogram. In National Guidelines for Thyroid Cancer Care and Treatment. Göteborg, Sweden: Regionalt Cancercentrum Väst.

Huang FW, Hodis E, Xu MJ, Kryukov GV, Chin L \& Garraway LA 2013 Highly recurrent TERT promoter mutations in human melanoma. Science 339 957-959. (https://doi.org/10.1126/science.1229259)

Huang DS, Wang Z, He XJ, Diplas BH, Yang R, Killela PJ, Meng Q, Ye ZY, Wang W, Jiang XT, et al. 2015 Recurrent TERT promoter mutations identified in a large-scale study of multiple tumour types are associated with increased TERT expression and telomerase activation. European Journal of Cancer 51 969-976. (https://doi.org/10.1016/j. ejca.2015.03.010)

Jendrzejewski J, Tomsic J, Lozanski G, Labanowska J, He H, Liyanarachchi S, Nagy R, Ringel MD, Kloos RT, Heerema NA, et al. 2011 Telomere length and telomerase reverse transcriptase gene copy number in patients with papillary thyroid carcinoma. Journal of Clinical Endocrinology and Metabolism 96 E1876-E1880. (https://doi. org/10.1210/jc.2011-1643)

Labourier E, Shifrin A, Busseniers AE, Lupo MA, Manganelli ML, Andruss B, Wylie D \& Beaudenon-Huibregtse S 2015 Molecular testing for miRNA, mRNA, and DNA on fine-needle aspiration improves the preoperative diagnosis of thyroid nodules with indeterminate cytology. Journal of Clinical Endocrinology and Metabolism 100 2743-2750. (https://doi.org/10.1210/jc.2015-1158)

Landa I, Ganly I, Chan TA, Mitsutake N, Matsuse M, Ibrahimpasic T, Ghossein RA \& Fagin JA 2013 Frequent somatic TERT promoter mutations in thyroid cancer: higher prevalence in advanced forms of the disease. Journal of Clinical Endocrinology and Metabolism 98 E1562-E1566. (https://doi.org/10.1210/jc.2013-2383)

Liu R \& Xing M 2016 TERT promoter mutations in thyroid cancer. Endocrine-Related Cancer 23 R143-R155. (https://doi.org/10.1530/ ERC-16-0206)
Liu X, Bishop J, Shan Y, Pai S, Liu D, Murugan AK, Sun H, El-Naggar AK \& Xing M 2013a Highly prevalent TERT promoter mutations in aggressive thyroid cancers. Endocrine-Related Cancer 20 603-610. (https://doi.org/10.1530/ERC-13-0210)

Liu Z, Li Q, Li K, Chen L, Li W, Hou M, Liu T, Yang J, Lindvall C, Bjorkholm M, et al. 2013b Telomerase reverse transcriptase promotes epithelial-mesenchymal transition and stem cell-like traits in cancer cells. Oncogene 32 4203-4213. (https://doi.org/10.1038/onc.2012.441)

Liu T, Wang N, Cao J, Sofiadis A, Dinets A, Zedenius J, Larsson C \& $\mathrm{Xu}$ D 2014 The age- and shorter telomere-dependent TERT promoter mutation in follicular thyroid cell-derived carcinomas. Oncogene 33 4978-4984. (https://doi.org/10.1038/onc.2013.446)

Liu T, Yuan X \& Xu D 2016 Cancer-specific telomerase reverse transcriptase (TERT) promoter mutations: biological and clinical implications. Genes 7 E38. (https://doi.org/10.3390/genes7070038)

Lloyd RV, Osamura RY, Klöppel G \& Rosai J 2017 WHO Classification of Tumours of Endocrine Organs WHO/IARC Classification of Tumours, 4th edn. Lyon, France: IARC Press.

Maruta J, Hashimoto H, Yamashita H, Noguchi H, Noguchi S, Kobayashi TK, Tsuneoka H \& Takahashi M 2015 Value of thyroid specific peroxidase and Ki-67 stains in preoperative cytology for thyroid follicular tumors. Diagnostic Cytopathology 43 202-209. (https://doi.org/10.1002/dc.23204)

Melo M, da Rocha AG, Vinagre J, Batista R, Peixoto J, Tavares C, Celestino R, Almeida A, Salgado C, Eloy C, et al. 2014 TERT promoter mutations are a major indicator of poor outcome in differentiated thyroid carcinomas. Journal of Clinical Endocrinology and Metabolism 99 E754-E765. (https://doi.org/10.1210/jc.20133734)

Nikiforov YE, Carty SE, Chiosea SI, Coyne C, Duvvuri U, Ferris RL, Gooding WE, LeBeau SO, Ohori NP, Seethala RR, et al. 2015 Impact of the multi-gene ThyroSeq next-generation sequencing assay on cancer diagnosis in thyroid nodules with atypia of undetermined significance/follicular lesion of undetermined significance cytology. Thyroid 25 1217-1223. (https://doi.org/10.1089/thy.2015.0305)

Saji M, Xydas S, Westra WH, Liang CK, Clark DP, Udelsman R, Umbricht CB, Sukumar S \& Zeiger MA 1999 Human telomerase reverse transcriptase (hTERT) gene expression in thyroid neoplasms. Clinical Cancer Research 5 1483-1489.

Shay JW \& Bacchetti S 1997 A survey of telomerase activity in human cancer. European Journal of Cancer 33 787-791. (https://doi. org/10.1016/S0959-8049(97)00062-2)

Tchirkov A, Chaleteix C, Magnac C, Vasconcelos Y, Davi F, Michel A, Kwiatkowski F, Tournilhac O, Dighiero G \& Travade P 2004 hTERT expression and prognosis in B-chronic lymphocytic leukemia. Annals of Oncology 15 1476-1480. (https://doi.org/10.1093/annonc/mdh389)

Vinagre J, Almeida A, Populo H, Batista R, Lyra J, Pinto V, Coelho R, Celestino R, Prazeres H, Lima L, et al. 2013 Frequency of TERT promoter mutations in human cancers. Nature Communication $\mathbf{4}$ 2185 .

Wang N, Liu T, Sofiadis A, Juhlin CC, Zedenius J, Hoog A, Larsson C \& $\mathrm{Xu}$ D 2014 TERT promoter mutation as an early genetic event activating telomerase in follicular thyroid adenoma (FTA) and atypical FTA. Cancer 120 2965-2979. (https://doi.org/10.1002/ cncr.28800)

Wang N, Kjellin H, Sofiadis A, Fotouhi O, Juhlin CC, Backdahl M, Zedenius J, Xu D, Lehtio J \& Larsson C 2016 Genetic and epigenetic background and protein expression profiles in relation to telomerase activation in medullary thyroid carcinoma. Oncotarget $\mathbf{7}$ 21332-21346.

Received in final form 5 April 2018

Accepted 24 April 2018

Accepted Preprint published online 24 April 2018 (c) 2018 Society for Endocrinology Published by Bioscientifica Ltd. Printed in Great Britain 